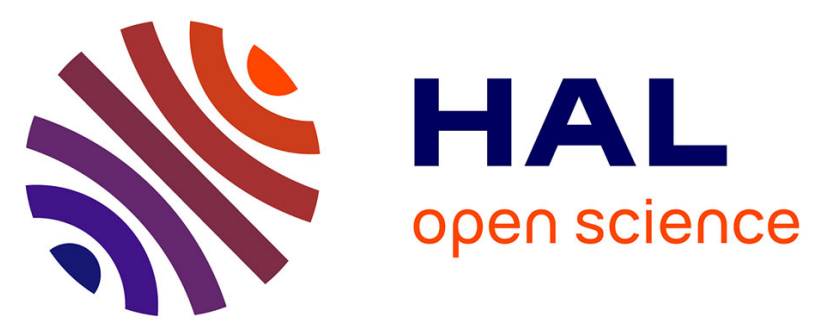

\title{
First Experimental Demonstration of Adaptive Optics Pre-Compensation for GEO Feeder Links in a Relevant Environment
}

\author{
Nicolas Védrenne, Aurélie Montmerle-Bonnefois, C B Lim, Cyril Petit, \\ Jean-François Sauvage, Serge Meimon, Philippe Perrault, Francis Mendez, \\ Bruno Fleury, Joseph Montri, et al.
}

\section{To cite this version:}

Nicolas Védrenne, Aurélie Montmerle-Bonnefois, C B Lim, Cyril Petit, Jean-François Sauvage, et al.. First Experimental Demonstration of Adaptive Optics Pre-Compensation for GEO Feeder Links in a Relevant Environment. 2019 IEEE International Conference on Space Optical Systems and Applications (ICSOS), Oct 2019, Portland, France. 10.1109/ICSOS45490.2019.8978970 . hal-02506508

\section{HAL Id: hal-02506508 \\ https://hal.science/hal-02506508}

Submitted on 30 Nov 2020

HAL is a multi-disciplinary open access archive for the deposit and dissemination of scientific research documents, whether they are published or not. The documents may come from teaching and research institutions in France or abroad, or from public or private research centers.
L'archive ouverte pluridisciplinaire $\mathbf{H A L}$, est destinée au dépôt et à la diffusion de documents scientifiques de niveau recherche, publiés ou non, émanant des établissements d'enseignement et de recherche français ou étrangers, des laboratoires publics ou privés. 


\title{
First experimental demonstration of adaptive optics pre-compensation for GEO feeder links in a relevant environment
}

\author{
$\underline{\text { N. Védrenne }}^{1}$, A. Montmerle-Bonnefois ${ }^{1}$, C.B. Lim $^{1}$, C. Petit ${ }^{1}$, J.-F. Sauvage $^{1}$, S. Meimon $^{1}$, P. Perrault ${ }^{1}$, \\ F. Mendez ${ }^{1}$, B. Fleury ${ }^{1}$, J. Montri ${ }^{1}$, J.-M. Conan ${ }^{1}$, V. Michau ${ }^{1}$, Z. Sodnik ${ }^{2}$, C. Voland ${ }^{2}$ \\ 1: High Angular Resolution Unit \\ ONERA \\ Châtillon, France \\ nicolas.vedrenne@onera.fr \\ 2: European Space Research and Technology Center, \\ ESA \\ Noordwijk, The Netherlands
}

\begin{abstract}
The future of very high throughput optical links between the ground and GEO satellites depends on the ability to overcome turbulence channel disruptions caused by the optical propagation through the atmosphere. Pre-compensation by adaptive optics has been identified as a game changer, as it could theoretically provide for the uplink the additional margin necessary to secure the link budget. Several experimental activities have been reported to investigate the practical increase that can be expected from $A O$ pre-compensation, demonstrating very promising results. Due to the proximity of the line of sight from ground and the strong variability of turbulence conditions these results would benefit from a confirmation on a more representative line of sight. The line of sight between ESA's Optical Ground Station and the top of Mount Teide provides an unprecedented opportunity to provide this confirmation in a relevant environment. We report here the results of the first experimental demonstration of uplink pre-compensation performed on this line of sight in April 2019. The increase of average optical power is demonstrated and compared to expected performance. The influence of anisoplanatism is demonstrated and quantified; consequences on the future of GEO feeder links are inferred.
\end{abstract}

Keywords—adaptive optics, anisoplanatism, ground station for optical feeder links.

\section{INTRODUCTION}

Link budget of uplink GEO feeder links is compromised by atmospheric turbulence influence. Adaptive optics (AO) has been identified as an enabling technology to mitigate channel impairments. Several experiments have been recently reported that demonstrated the technology feasibility for pre-compensation. These demonstrations took place either on a short path [1] or on a $10 \mathrm{~km}$ long horizontal path [2]. In both cases the representativeness of the line of sight is limited. Short distance implies limited turbulence strength; horizontal path induces strong perturbations that will affect wavefront sensing performance and the relevancy of precompensation efficiency. As a consequence, adaptive optics compensation still has to be demonstrated in representative turbulent environment. Although analytical models are available for quantifying the effect of point ahead angle (PAA) anisoplanatism on ground-space optical links (see [3] and references therein), an experimental assessment of its impact on the link budget remains of great interest.

The FEEDEr LInk adaptive Optics (FEEDELIO) project aimed at demonstrating the feasibility of AO pre- compensation in a relevant environment and to assess expected performance for variable turbulent conditions in the presence of anisoplanatism.

\section{FEEDELIO EXPERIMENT}

\section{A. Overview of the experimental setup}

The FEEDELIO experiment took place in Tenerife during the two first weeks of April 2019 on a $13.2 \mathrm{~km} 5^{\circ}$ elevation slant path between ESA's optical ground station (OGS) and top of mount Teide [4].

The hardware block diagram of the experiment setup, already presented in [5][6] is recalled Figure 1.

To provide the adaptive optics reference beam and the detection of the uplink pre-compensated beam a satellite terminal breadboard (STB) emulator was attached to the concrete wall of the cable car accommodation building.

To provide the uplink pre-compensated beam a ground terminal breadboard (GTB) was attached on the south pillar of ESA's OGS.

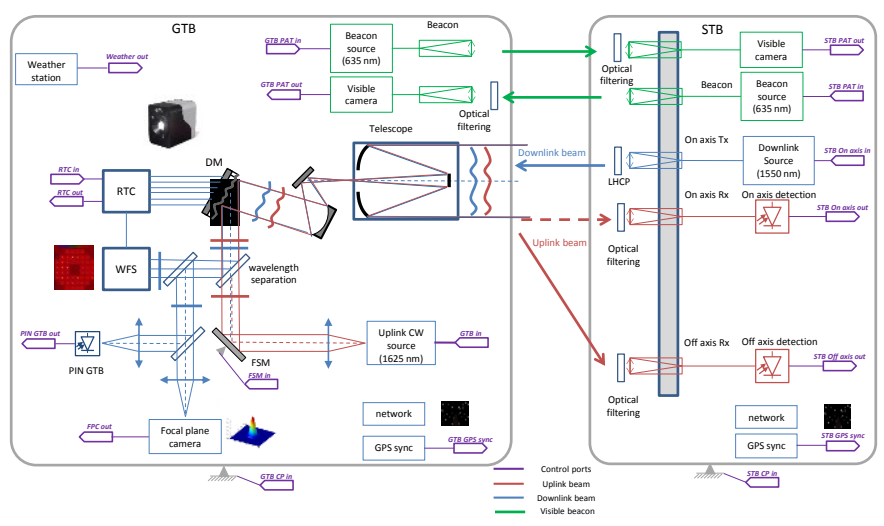

Figure 1 - Hardware block diagram of the FEEDELIO experiment.

\section{B. Satellite terminal breadboard}

The STB was providing the $1550 \mathrm{~nm}$ reference beacon for wavefront sensing and the uplink detection. Uplink detection was performed through two multimode fibers coupled $1.7 \mathrm{~mm}$ diameter collimators sending the light on a $20 \mathrm{kHz}$ low noise PIN detectors installed in two separate modules: one on axis module also providing the reference 
beacon, and one off axis movable module. In order to investigate the influence of point ahead angle, the off axis module was fixed on a translating platform remotely controlled hence providing the possibility to investigate a range from $3 \mu \mathrm{rad}$ up to $100 \mu \mathrm{rad}$ angular excursion.

\section{Ground terminal breadboard}

The GTB was composed of a $350 \mathrm{~mm}$ commercial telescope and of an optical breadboard carrying the adaptive optics system, the downlink measurement path and the uplink $1625 \mathrm{~nm}$ source. In order to cope with the evolution of turbulent conditions during the experiment the acquisition protocol was designed to address alternatively on and off axis module within a limited duration thanks to the use of a dedicated fast steering mirror on the uplink path. A 10s time period has been used here. In this timeframe turbulence conditions (Fried parameter and scintillation index) has proven to be stable enough with respect to AO error budget.

\section{Adaptive optics}

The adaptive optics system has been built from commercial off the shelf components: the deformable mirror was an Alpao 95-17 (fast upgrade), the wavefront sensor camera was a Raptor Owl HS, and the real-time computer was provided by Shakti upon ONERA's requirements. The wavefront sensor counted $8 \times 8$ subapertures and was used at a $1.5 \mathrm{kHz}$ frequency providing a 2,3 frame delay. The adaptive optics system has been designed to provide a $2.2 \mathrm{~dB}(0,6$ attenuation) average coupling loss (wrt diffraction limit) for $\mathrm{r}_{0}=11 \mathrm{~cm}$. Thanks to the large stroke of the deformable mirror no dedicated tip/tilt mirror has been used. A motorization of the deformable mirror mount was only used to cope with refraction index evolution encountered during daytime. More details on the AO loop performance for downlink are available in [7]. We focus here on the uplink results.

\section{EXPERIMENT RESULTS}

\section{A. Data acquisitions}

Due to very poor weather conditions during the first week of the experiment (access to cable car closed), STB could only be installed at the very beginning of the second week of experiment. Experiment calibration and adjustments took 3 days, leaving four days for data acquisitions. We focus here on the last day of acquisitions (14/04/19). During one set of acquisitions uplink power measured on both on-axis and offaxis modules has been recorded simultaneously with a synchronized protocol. AO loop telemetry (wavefront slopes, voltages sent to the deformable mirror and intensity per subaperture) has been recorded jointly.

\section{B. Turbulence parameters estimation}

Wavefront sensor measurements are exploited to estimate optical turbulence conditions. A Fried parameter $\left(\mathrm{r}_{0}\right)$ estimate is obtained from a fit of the Zernike's spatial variance with Noll's power law [8], downlink power fluctuations are computed from Shack-Hartmann wavefront sensor (SHWFS) intensity measurements. By exploiting the spatial correlation of slopes and intensity measurements, assuming small perturbations as described in [9], a $\mathrm{C}_{\mathrm{n}}{ }^{2}$ profile estimate can be computed for every $5 \mathrm{~s}$ time slot. This $\mathrm{C}_{\mathrm{n}}{ }^{2}$ profile can be used to compute analytically another estimate of $\mathrm{r}_{0}$ according to [10] and of power fluctuations normalized variance in a given square subaperture $\sigma^{2}{ }_{i}$ according to aperture averaged scintillation theory [11][12]. Turbulence profile estimation also enables the computation of an isoplanatic angle estimate [13]. All these integrated parameters estimations are performed while taking into account the sphericity of the incoming beam from the STB module.

The results of this turbulent profile estimation are presented in Figure 2. The top of the figure presents $r_{0}$ estimation from SHWFS data (obtained from Noll's fit) in black and the result of $\mathrm{r}_{0}$ estimation from the estimated $\mathrm{C}_{\mathrm{n}}{ }^{2}$ as a function of time. The center of the figure presents $\sigma^{2}{ }_{i}$ measured by the SHWFS in black and its estimation from the $\mathrm{C}_{\mathrm{n}}{ }^{2}$ profile in blue.

Apart from the cases where the smooth perturbation approximation is obviously not satisfied (outside the Rytov regime, corresponding to log-amplitude variance above 0.3 ie $\left.\sigma^{2}{ }_{i}>1.2\right)$ the turbulence profile estimate provides a reliable evaluation of $r_{0}$ and $\sigma^{2}{ }_{i}$ hence a good confidence in the estimation of the isoplanatic angle $\theta_{0}$.

In the following, the study is restricted to turbulence cases within the smooth perturbation approximation. Considering the elevations expected from future GEO-feeder links, above $30^{\circ}$ in European area, this hypothesis shall be satisfied.

As illustrated here a wide variability of anisoplanatism conditions has been encountered, with isoplanatic angle $\theta_{0}$ values ranging from $25 \mu \mathrm{rad}$ down to less than $5 \mu \mathrm{rad}$.
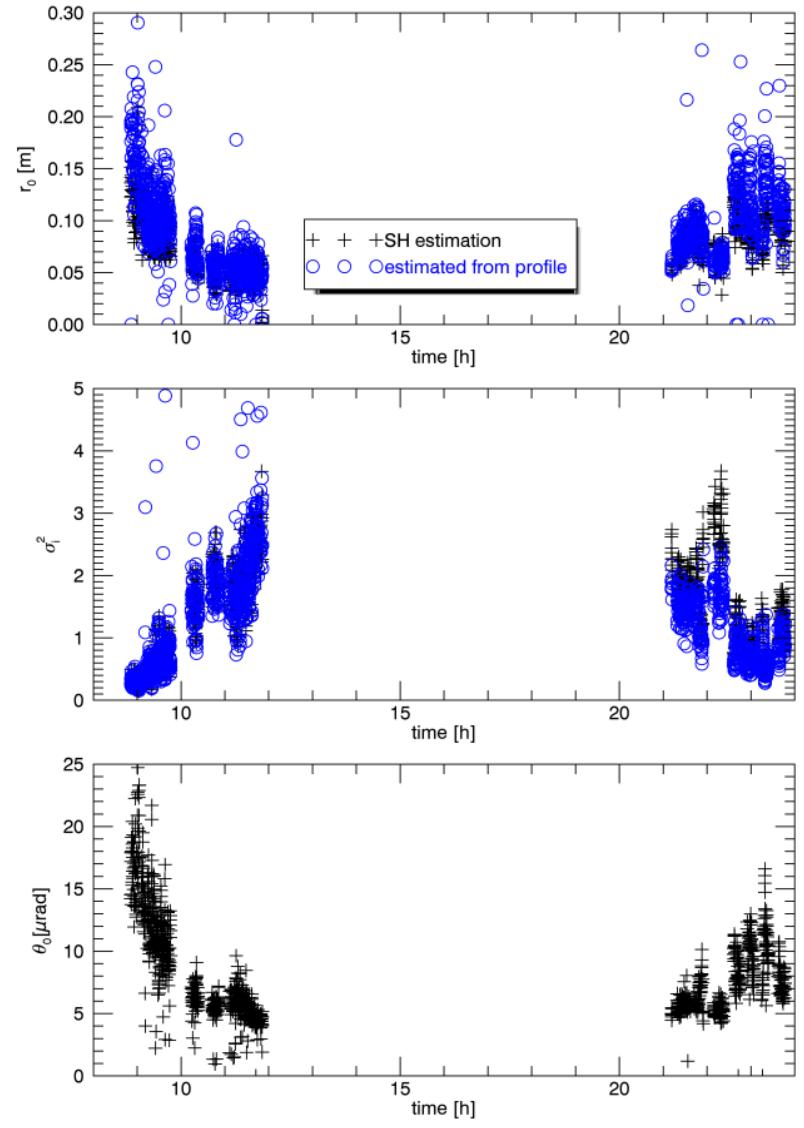

Figure 2 - Top: Fried parameter estimated from ShackHartmann measurement (black) and from turbulence profile estimation (blue). Center: flux fluctuations variance per subaperture from Shack-Hartmann measurements (black) and estimated from turbulence profile (blue). Bottom: isoplanatic angle estimated from turbulence profile. 
C. Anisoplanatism influence and turbulence conditions variability
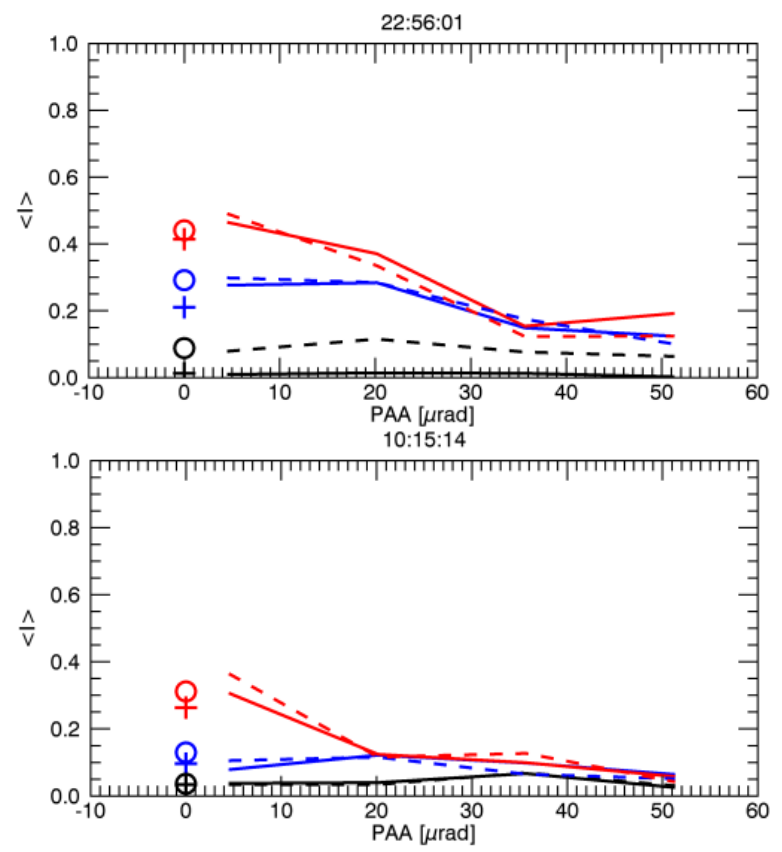

Figure 3 - Evolution of the average coupling wrt diffraction limit as a function of the PAA, top for a small turbulence case $\left(r_{0}=10 \mathrm{~cm}, \theta_{0}=10 \mu \mathrm{rad}\right)$, bottom for the stronger turbulence case $\left(r_{0}=5 \mathrm{~cm}, \theta_{0}=6 \mu \mathrm{rad}\right)$. Continuous line: off-axis experiment, dashed line: offaxis theory. Crosses: on axis measurements; rounds: on axis theory. Red: full correction; blue: tip/tilt only; black: no correction.

We illustrate here for two cases the influence of anisoplanatism on the correction efficiency obtained during the experiment. We plot on Figure 3 the average coupling efficiency $<$ I $>$ of the flux in the receiver with respect to the geometrical losses (diffraction limited beam corresponds to $<$ I $>=0.74$ due to the uplink central obscuration). Two cases corresponding to different perturbation strength are illustrated: a favorable case, obtained by nighttime (22:56 p.m.), with $\mathrm{r}_{0}=10 \mathrm{~cm}, \theta_{0}=10 \mu \mathrm{rad}$; a more perturbed case, obtained by daytime, around 10:15 a.m., with $\mathrm{r}_{0}=5 \mathrm{~cm}, \theta_{0}=$ $6 \mu \mathrm{rad}$.

To cope with potential turbulence non-stationarity both onaxis and off-axis measurements are performed alternatively with a 10s time period. We investigate four off-axis positions, each of them corresponding also to an on-axis measurement. We plotted the average of the four on-axis measurements (crosses) performed during the acquisition of the off-axis measurements and theoretical model results (round symbols) for 3 correction cases : red for a correction of all the degrees of freedom enabled by the deformable mirror, blue for a tip/tilt only correction, and without correction in black. Off axis measurements as a function of the point ahead angle are also reported: continuous lines correspond to measurements, dashed lines to theoretical performance models while taking into account the estimated turbulent profile.

Anisoplanatism influence appears significantly different for both cases: by daytime, angular decorrelation of the perturbations induces a steep decrease of $<\mathrm{I}>$ as a function of the PAA. By nighttime the correction degradation is much more progressive.

For both cases the theoretical model (see [3] and references therein), not described here, adequately reproduces the measurement for on-axis and off axis cases.

\section{Transposition to a GEO-feeder link case.}

We analyze here the contributors to the error budget affecting off axis performance. We first study the on-axis measurements and compare them to theoretical evaluation to assess the on-axis error budget. Then we consider the offaxis terms for a PAA value representative of GEO-feeder link anisoplanatism conditions and compare it to theory.

In the presence of anisoplanatism (hence for off-axis measurement) the theoretical average power penalty term attributed to the correction of atmospheric effects can be written in a first approximation as:

$<I>_{\text {aniso }} \approx \rho_{0} \exp \left[-\sigma_{\text {fitting }}{ }^{2}-\sigma_{\text {scintillation }}{ }^{2}-\sigma_{\text {aniso }}{ }^{2}(P A A)\right]$

In Equation (1), $\rho_{0}$ is the diffraction limited coupling efficiency for a $23 \%$ obscuration antenna $\left(\rho_{0}=0.74\right.$ according to [14]), $\sigma_{\text {fitting }}{ }^{2}$ stands for the fitting error term computed as follows [15]:

$$
\sigma_{\text {fitting }}{ }^{2}=0.458\left(n_{r}+1\right)^{-5 / 3}\left(D / r_{0}\right)^{5 / 3}
$$

$\sigma_{\text {scinitilation }}{ }^{2}$ designates the variance of $\log$ amplitude fluctuations affecting coupling efficiency, it is approximated here, assuming the small perturbation approximation and neglecting the influence of aperture averaging at the scale of a subaperture, by $\sigma_{i}^{2} / 4$; while $\sigma_{\text {aniso }}{ }^{2}(P A A)$ designates the anisoplanatism error contribution at PAA, and can be computed according to [16].

The ultimate limit to AO performance is fixed by the fitting error term. Its contribution is plotted in Figure 4 in dashed black lines. The on-axis measurements are plotted with black crosses. In order to limit the dispersion of the data plotted, measured values have been averaged over $1 \mathrm{~cm}$ bins in $r_{0}$. Error bars correspond to the standard deviation of the values averaged on a $1 \mathrm{~cm} \quad \mathrm{r}_{0}$ slot (when several measurements are available).

Note that measurements have been normalized in units consistent with the theoretical average coupling efficiency defined by Eq. (1).

Clearly on-axis measurements are smaller than fitting only performance. Adding the scintillation term of Eq. (1) however leads to a good fit of on-axis data.

The choice of the FEEDELIO off-axis angle giving a relevant GEO-Feeder PAA emulation is a delicate question. Two options are discussed in [5].

The most relevant, that we will call further the modal approach is to consider an off-axis angle leading to a performance degradation, compared to on-axis, identical to the one expected for similar conditions in the case of GEOfeeder link. This implies a fine modeling of the anisoplanatism error accounting for the number of corrected modes and the turbulence profile. This is out of the scope of this paper.

Another possibility is to consider a more straightforward but somewhat unprecise approach based on the conservation of the ratio between PAA and isoplanatic patch $\theta_{0}$. The PAA for a GEO-feeder link is $18 \mu \mathrm{rad}$. According to recently 
published statistics in Cerro-Paranal [17] median isoplanatic patch for such an observatory-like location is $\theta_{0}=10 \mu \mathrm{rad}$. Without any exhaustive documentation of this quantity around the globe, considering such a value as representative might be questionable. Still, based on these elements one would want to conserve the ratio PAA $/ \theta_{0}=1.8$ is. As exposed in [5], this simple approach could however lead to a (very) pessimistic estimate of anisoplanatism compared to the modal approach. Hence the trends identified in this case must be considered cautiously. Based on the analysis given in [5], a reasonable range from the equivalent PAA could be between $\mathrm{PAA}_{\text {equivalent }}=\theta_{0}$ and $\mathrm{PAA}_{\text {equivalent }}=1.8 \theta_{0}$. Results obtained from the experiment in these two cases together with their theoretical evaluation based on the estimated profile are plotted Figure 4 as a function of the encountered $r_{0}$. They can be compared to on-axis tip/tilt only correction measurements (black crosses). These data points are measured on-axis but we remind that tip/tilt correction at PAA is barely affected by anisoplanatism [5]. This is confirmed by Figure 3: tip/tilt corrected results being constant in the range $\mathrm{PAA}=0$ and $\mathrm{PAA}=1.8 \theta_{0}$.

The analysis of the experimental results demonstrate the interest for high order pre-compensation in the presence of anisoplanatism, as PAA affected measurements are systematically above tip/tilt only correction. Besides, theoretical performance evaluation from anisoplanatism models accurately fit the off-axis measurements. Assuming the relevant equivalent $\mathrm{PAA}$ is between $\mathrm{PAA}_{\text {equivalent }}=\theta_{0}$ and $\mathrm{PAA}_{\text {equivalent }}=1.8 \theta_{0}$, the gain wrt tip/tilt only correction shall be between $1 \mathrm{~dB}$ and $2 \mathrm{~dB}$ depending on the turbulence profile.

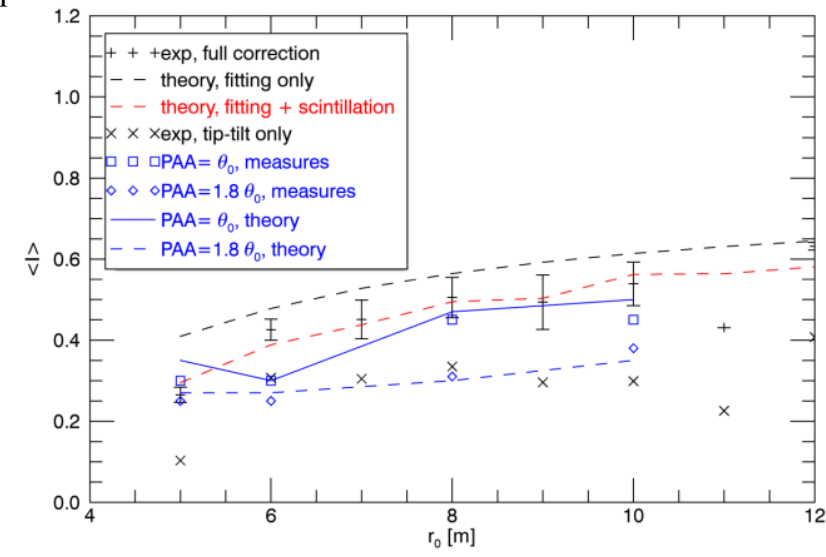

Figure 4 - Average coupling on the STB side as a function of $\mathbf{r}_{0}$. Black "+": measurements with all possibly corrected degrees of freedom, dashed lines: theoretical evaluation with fitting error term only; red dashed lines: theoretical evaluation including fitting and scintillation influence. Black crosses: on-axis tip/tilt correction only measurements; blue crosses: average coupling for $\mathbf{P A A}=$ $1.8 \theta_{0}$ (measures), dashed blue line: average coupling for PAA $=1.8 \theta_{0}$ (experiment)

\section{E. Discussion}

The analysis provided here has only been based on average power losses encountered on a single day of acquisition. In order to consolidate the data analysis, deeper investigations are therefore necessary: evaluation of power statistics (mean and fluctuations) in a wide range of turbulence conditions, precise evaluation of the equivalent PAA based on the modal approach. The temporal signature of power losses could also be studied and compared to the GEO-Feeder case.

With respect to average power penalty, for the turbulence conditions encountered here, results obtained by precompensation led to a few $\mathrm{dB}$ gain compared to tip/tilt only correction. This may seem small but one has to remember that AO not only increases the average detected flux but also drastically reduces flux fluctuations and budget link gains can be shown to be mainly driven from this latter effect. Based on numerical modeling, higher order correction indeed gives few $\mathrm{dB}$ gains on average flux, compared to tiptilt, but gain is much more substantial (often more than $10 \mathrm{~dB}$ ) in link availability thresholds Erreur ! Source du renvoi introuvable.

PAA anisoplanatism is still a strong limiting factor especially when going to large aperture (few $10 \mathrm{~cm}$ ) emitters [3]. To truly benefit from the Tx full antenna gain one needs to find ways to reduce this anisoplanatism effect. Several strategies can be considered to circumvent PAA influence. Among others the design of appropriate control laws or the use of specific configurations of laser guide stars are already investigated. The most drastic solution is probably the concept suggested by Tyson in 1996[18], ie the use of a free flyer support satellite to provide the beacon in the direction of the PAA.

\section{CONCLUSION}

We reported here the results of the first experimental demonstration of pre-compensation for GEO - feeder links in a relevant environment. The experiment took place in Tenerife April 2019 on a $13.2 \mathrm{~km}$ slant path line of sight between ESA's OGS and top of Mount Teide in Tenerife. Due to ground proximity, strong turbulence perturbations conditions were encountered. Restricting the analysis to smooth perturbations case, more relevant for GEO-feeder links, the feasibility of a pre-compensation by adaptive optics has been confirmed. Performance models to estimate the average power penalty fit well with measurements. The influence of PAA has been quantified experimentally and compared successfully to anisoplanatism error evaluation. The question of the off-axis angle equivalent to GEO-feeder PAA is discussed. Depending on the assumptions taken for this equivalence, gains in average power brought by correction beyond tip-tilt vary but are systematic. Besides, link budget has to account both for average power and statistical distributions. A systematic analysis of the collected data statistics is therefore now required. Particular interest shall also be granted to temporal signature of the power fluctuations. In any case, anisoplanatism highly restricts the potential gain of $\mathrm{AO}$ with significant size telescopes (few tens of $\mathrm{cm}$ ). Appropriate strategies must be investigated to circumvent anisoplanatism limitation. Among others, the use of a free flyer satellite to provide the beacon for wavefront sensing in the direction of the PAA is conceptually the most promising one.

\section{ACKNOWLEDGMENT}

This work has been performed in the framework of ESA's contract No4000120300/17/NL/PS. FEEDELIO experiment could not have been possible without the very 
helpful contributions of the technical staff from Instituto Astrofísica de Canarias, Teide's National Park and Teide's cable car station. The author warmly thanks them for their fruitful help and collaboration.

\section{REFERENCES}

[1] A. Brady et al., "Experimental validation of phase-only precompensation over $494 \mathrm{~m}$ free-space propagation," Opt. Lett., vol. 42 , no. 14, pp. 2679-2682, Jul. 2017.

[2] R. Saathof et al., "Pre-correction adaptive optics performance for a 10 km laser link," in Free-Space Laser Communications XXXI, 2019, vol. 10910, p. $109101 \mathrm{H}$.

[3] J.-M. Conan, N. Védrenne, A. Montmerle-Bonnefois, C. Petit, M.-T. Velluet, C. Robert, C. B. Lim, V. Michau, G. Artaud \& B. Benammar, "Adaptive optics for ground - GEO-satellites optical links: from system design to experimental demonstration," Proc. of AOIM XII Conference, in press (2019).

[4] A. Montmerle-Bonnefois et al., "Adaptive optics precompensation of a GEO feeder link: the FEEDELIO experiment," In Appli. of Lasers for Sensing and Free Space Comm. (pp. LTh1B-3), Optical Society of America, (2019).

[5] N. Védrenne, J. Conan, A. Bonnefois, C. Petit, M. Velluet, and V. Michau, "Adaptive optics pre-compensation for GEO feeder links: Towards an experimental demonstration," in 2017 IEEE International Conference on Space Optical Systems and Applications (ICSOS), 2017, pp. 77-81.

[6] A. Montmerle Bonnefois et al., "Adaptive optics pre-compensation for GEO feeder links: the FEEDELIO experiment," in Proc. SPIE 11180, ICSO 2018 (2019).

[7] C. B. Lim et al., "Single-mode fiber coupling with adaptive optics for free-space optical communication under strong scintillation," IEEE
International Conference on Space Optical Systems and Application, Portland, Oregon, USA (2019).

[8] R. J. Noll, "Zernike polynomials and atmospheric turbulence," J Opt Soc Am, vol. 66, no. 3, pp. 207-211, 1976.

[9] N. Védrenne, A. B. Montmerle, C. Robert, V. Michau, J. Montri, and B. Fleury, " $\mathrm{C}_{\mathrm{n}}{ }^{2}$ profile measurement from Shack-Hartmann data: experimental validation and exploitation," in Optics in Atmospheric Propagation and Adaptive Systems XIII, 2010, vol. 7828, p. 78280B.

[10] D. L. Fried, "Statistics of a Geometric Representation of Wavefront Distortion," J. Opt. Soc. Am., JOSA, vol. 55, no. 11, pp. 1427-1435, Nov. 1965.

[11] D. L. Fried, "Aperture Averaging of Scintillation," J. Opt. Soc. Am., vol. 57, no. 2, p. 169, Feb. 1967.

[12] J. H. Churnside, "Aperture averaging of optical scintillations in the turbulent atmosphere," Appl. Opt., AO, vol. 30, no. 15, pp. 19821994, May 1991.

[13] D. L. Fried, "Anisoplanatism in adaptive optics," J. Opt. Soc. Am., JOSA, vol. 72, no. 1, pp. 52-61, Jan. 1982.

[14] B. J. Klein and J. J. Degnan, “Optical Antenna Gain. 1: Transmitting Antennas,” Appl. Opt., AO, vol. 13, no. 9, pp. 2134-2141, Sep. 1974.

[15] J.-M. Conan, "Etude de la correction partielle en optique adaptative," thesis, Paris 11, 1994.

[16] F. Chassat, "Propagation optique a travers la turbulence atmospherique: etude modale de l'anisoplanetisme et application a l'optique adaptative," thesis, Paris 11, 1992.

[17] J. Osborn and M. Sarazin, "Atmospheric turbulence forecasting with a general circulation model for Cerro Paranal," Monthly Notices of the Royal Astronomical Society, vol. 480, no. 1, pp. 1278-1299, Oct. 2018.

[18] R. K. Tyson, "Adaptive optics and ground-to-space laser communications," Appl. Opt., AO, vol. 35, no. 19, pp. 3640-3646, Jul. 1996. 